$>$ La COVID-19 est une infection pandémique émergente dont l'importante capacité à se propager dans une population dénuée d'immunité n'est plus à prouver. La première réponse des États à la flambée de COVID-19 fut d'imposer un confinement et des mesures barrières, telles que le port du masque et la distanciation sociale. Une des répercussions de cette pandémie, en matière de santé publique, fut la suspension ou le ralentissement brusque des campagnes de vaccination des nourrissons, un peu partout dans le monde. Un des effets indirects de la COVID-19 est donc le risque de peser sur la mortalité mondiale, principalement via une recrudescence de la rougeole et de la poliomyélite, principalement dans les pays en voie de développement. Dans ce chaos potentiel, le seul espoir réside dans le développement rapide d'un vaccin efficace contre le SARS-CoV-2 (severe acute respiratory syndrome-coronavirus-2). Cependant, I'acceptation de ce vaccin par la population n'est pas évidente, car outre les nombreuses inconnues qui vont peser inévitablement dans le cas d'un développement très rapide du vaccin, le scepticisme des hésitants vaccinaux va à nouveau se développer.<

La coronavirus disease (COVID-19) causée par le severe acute respiratory syndrome-coronavirus type 2 (SARSCoV-2) poursuit ses effets dévastateurs autour du monde. Elle revient en Europe, après une brève trêve gagnée laborieusement à la faveur d'un confinement global et inédit de plus de deux mois en France. Au 16 octobre 2020, le site de la Johns Hopkins University ${ }^{1}$ répertoriait officiellement près de 39 millions de cas et plus de 1,1 million de morts dans le monde, alors que

Vignette (Photo @ Philippe Roingeard et Sébastien Eymieux, Université de Tours, France).

https://coronavirus.jhu.edu/map.htm

\section{COVID-19 \\ et vaccination : \\ une dérégulation globale}

\author{
Emmanuelle Billon-Denis ${ }^{1,2}$, \\ Jean-Nicolas Tournier ${ }^{1-3}$
}

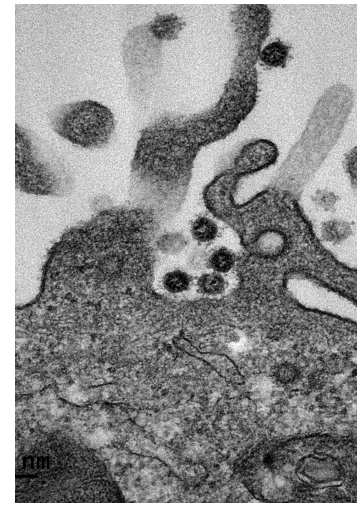

${ }^{1}$ Institut de recherche biomédicale des armées (IRBA), Unité biothérapies anti-infectieuses et immunité, 1 place Général Valérie André, 91220 Brétigny-sur-Orge, France. ${ }^{2}$ Institut Pasteur, Innovative vaccine laboratory, 75015 Paris, France.

${ }^{3}$ École du Val-de-Grâce, 1 place Alphonse Laveran, 75005 Paris, France.

jean-nicolas.tournier@intradef. gouv.fr

jean-nicolas.tournier@pasteur.fr

la situation dans la zone Amérique ne fait qu'empirer, que les données du continent africain ne sont que partiellement connues, et que l'Europe connaît une deuxième flambée épidémique. Les répercussions de la COVID-19 sur la santé globale ne sont pas uniquement lisibles à travers ce bilan direct de mortalité liée au virus. La COVID-19 a des effets indirects dérégulateurs sur l'état de santé des populations en raison de son impact sociétal, économique et géopolitique global.

Cet article fait un point sur les données des effets de la COVID-19 sur la vaccination des enfants et adolescents en France et dans le monde, et sur les perspectives probables en termes de vaccination, avec l'arrivée de nouveaux vaccins annoncée pour la fin 2020.

\section{La réponse à l'épidémie et la vaccination en France}

La brutalité de l'épidémie et de la mise en place du confinement du mardi 17 mars à midi au lundi 11 mai 2020 au matin a eu des répercussions importantes sur la vaccination des jeunes enfants en France. Dans un avis du $1^{\text {er }}$ avril 2020, la Haute autorité de santé (HAS) a rappelé l'importance de la vaccination des nourrissons dès le $2^{\mathrm{e}}$ mois, et du maintien d'un haut taux de couverture vaccinale [1]. Cet appel a été repris par l'Académie nationale de médecine dans un avis du 28 avril 2020 [2]. Le Groupement d'intérêt scientifique (GIS) EPI-PHARE, constitué fin 2018 par la caisse nationale d'assurance maladie (CNAM) et l'agence nationale de sécurité des médicaments et des produits de santé (ANSM), révèle que les vaccins penta- et hexavalents destinés au nourrisson ont subi une baisse de délivrance de $35 \%$ au cours de cette période de confinement, et que les délivrances des vaccins contre la rougeole, les oreillons et la rubéole (ROR) et 
contre les human papilloma virus (HPV) ont chuté, respectivement de $43 \%$ et $67 \%$, au cours de la deuxième semaine d'avril 2020 [3]. À la même date, les ventes de vaccins antitétaniques ont chuté de $71 \%$. En valeur absolue, plus de 77000 nourrissons, âgés de 3 à 18 mois, n'ont pas été vaccinés durant les 5 semaines de confinement, ceci pour les vaccins hexavalents. À la sortie du confinement, plus de 93000 nourrissons n'avaient pas reçu le vaccin trivalent ROR (rougeole-oreillonsrubéole) ; 285000 enfants, adolescents et adultes étaient en attente d'un rappel antitétanique ; et 59000 adolescents n'avaient pas reçu leurs vaccins anti-HPV. Les effets à long terme de cette désorganisation dans l'accès à la vaccination apparaissent éventuellement longs et incertains à rattraper.

\section{Les campagnes de vaccination dans le monde}

Dans les pays en voie de développement, l'épidémie de COVID-19 a désorganisé des systèmes de santé déjà fragiles, pour lesquels le fonds des Nations-unies pour l'enfance (UNICEF) est le principal pourvoyeur de vaccins. En Afrique de l'ouest, l'épidémie d'infections par le virus Ebola, qui a sévi entre 2014 et 2015 , avait déjà complètement désorganisé la lutte contre le paludisme et la vaccination contre la rougeole [4] : dans les suites de l'épidémie, deux fois plus d'enfants étaient morts de rougeole que d'infection par le virus Ebola, en raison de la suspension des campagnes de vaccination contre la rougeole des populations habituellement ciblées [5]. Alors que la COVID-19 épargne les enfants, la résurgence de deux maladies prévenues par une vaccination sont ainsi plus particulièrement craintes dans les pays en voie de développement en raison de l'absence d'accès aux vaccins : la rougeole et la poliomyélite.

\section{La poliomyélite}

La poliomyélite est une maladie en voie d'éradication causée par un entérovirus dont il existe trois sérotypes [6]. Le sérotype 2 n'est plus observé depuis 1999, et le sérotype 3 depuis 2012 ; ces deux sérotypes sont désormais déclarés comme éradiqués. En 2020, deux pays déclarent encore des cas d'infection par le virus de la poliomyélite sauvage de type 1 : le Pakistan et l'Afghanistan. Les données des campagnes récentes de vaccination par le vaccin oral contre la poliomyélite au Pakistan montrent un taux de refus de $20 \%$, suggérant des difficultés futures à atteindre les populations cibles [5]. Les efforts de I'Organisation mondiale pour la santé (OMS) tendaient à une réduction des cas à la fin de la décennie passée, avec 57 cas d'infection par le poliovirus de type 1 répertoriés sur toute l'année 2019. Malheureusement, l'année 2020 inverse la tendance globale avec 70 cas déjà certifiés sur le seul premier semestre 2020. Les risques d'un retour explosif de la poliomyélite ne sont donc pas à exclure, même sur le continent africain, qui a pourtant réussi à l'éradiquer ${ }^{2}[7]$.

\footnotetext{
${ }^{2}$ Un cas récent vient d'être cependant signalé fin août 2020 dans le Sud Soudan.
}

\section{La rougeole}

En mars 2020, l'OMS a suspendu ses campagnes de vaccination de masse contre la rougeole afin de limiter la diffusion du SARS-CoV-2 [8] avec, pour conséquence, plus de 120 millions d'enfants qui risquent de ne pas avoir été vaccinés contre la rougeole [9]. Un travail de modélisation a proposé, dans une projection qualifiée de «moins sévère », qu'une diminution des campagnes de vaccination contre la rougeole de $15 \%$ pendant 6 mois entraînerait le décès de 253500 enfants et 12200 décès maternels [10]. En République démocratique du Congo, la COVID-19 avait tué en juin 2020, moins de 37 personnes. En comparaison, l'année 2019 a vu la rougeole toucher 310000 personnes, principalement des enfants, et faire plus de 6000 victimes [9]. Ces chiffres montrent à quel point la peur de l'infection et la dérégulation des systèmes de soins qui se sont orientés subitement sur la prévention de la COVID-19 peuvent avoir des effets meurtriers bien supérieurs chez les enfants, notamment en Afrique dont les populations sont très jeunes.

\section{Le futur: des annonces complexes au sujet de la vaccination contre le SARS-CoV-2 et une acceptation vaccinale générale difficile}

Le nombre de projets de vaccins dirigés contre le SARS-CoV-2 en développement augmente actuellement de manière exponentielle. Plus de 321 projets sont aujourd'hui recensés, pour 120 projets dénombrés au mois d'avril 2020, soit une augmentation de plus de 2,5 fois en 4 mois [11]. Parmi ces projets, 32 candidats vaccins sont en cours d'essais cliniques, avec des objectifs de recrutement de plus de 280000 participants provenant d'au moins 470 centres d'inclusion dans 34 pays différents. Au $1^{\text {er }}$ octobre 2020, au moins six laboratoires avaient publié des résultats d'essais de phase I et II de vaccins dirigés contre le SARS-CoV-2, avec la promesse de l'induction d'une immunité au moins immédiate et, chez l'homme, la production d'anticorps neutralisants. Trois essais portaient sur des plateformes vaccinale adénovirus : les laboratoires de l'Institut Jenner de l'université d'Oxford au Royaume-Uni, soutenus par AstraZenaca, avec un vaccin fondé sur l'utilisation d'un adénovirus de chimpanzé ChAd0xl nCoV19 [12]; CanSino Biologics à Wuhan en République populaire de Chine utilisant un adénovirus de type 5 non réplicatif [13] ; et en Fédération de Russie, l'approche du vaccin «Spoutnik V » de l'Institut Gamaleya annoncée directement par le président russe début août 2020 avec un vaccin non réplicatif d'adénovirus humains de type 26 et de type 5 utilisés en stratégie «prime-boost » (avec 
nécessité d'un rappel) [14]. Depuis, des suspicions de fraudes ont été émises sur les résultats publiés par l'institut Gamaleya, laissant planer des doutes sur la fiabilité des résultats [15]. Un essai développé par Novavax aux États-Unis qui reposait sur l'utilisation de la protéine Spike virale native, sous forme recombinante et comprenant le domaine transmembranaire de la protéine associé à des nanoparticules, et à un adjuvant à base de saponine, appelé Matrix Ml, a aussi donné une immunité dite neutralisante (car induisant des anticorps neutralisant le virus) [16].

Enfin, aux États-Unis également, une approche très novatrice développée par la firme Moderna portait sur un vaccin à base de I'ARNml273 codant la protéine $S$ du virus $[17,18](\rightarrow)$. En $(\rightarrow)$ Voir la Nouvelle de B. Pitard, $\mathrm{m} / \mathrm{s}$ $n^{\circ} 10$, octobre 2019 , page 749
Allemagne, l'université de Mainz, avec le soutien du laboratoire Pfizer, a testé différentes doses de l'ARNm BNTl62bl encapsidé dans des lipides codant la protéine $S$ du virus dans un essai de phase I/II. Cette stratégie semble induire une immunité humorale et cellulaire [19, 20]. Ces six approches sont toutes fondées sur l'induction d'une immunité dirigée contre la glycoprotéine de surface Spike du SARS-CoV-2, comme la majorité des vaccins actuellement en développement.

$\varepsilon n$ plus de ces résultats encourageants concernant l'induction d'une réponse immunitaire potentiellement protectrice [21], les efforts en recherche et développement ne tarissent pas puisque tous les grands acteurs en matière de production de vaccins annoncent des travaux dans ce domaine hautement concurrentiel. Les candidats vaccins validés en phase I/II sont tous entrés en phase III d'essais cliniques de validation de leur efficacité protectrice et les fabricants de vaccins se préparent tous à une production vaccinale de masse. Le plus grand fabricant de vaccins au monde, le Serum Institute of India à Pune, a conclu un accord pour fabriquer un milliard de doses d'un vaccin contre le coronavirus développé par AstraZeneca, s'il est approuvé pour son utilisation. Le 9 septembre 2020, AstraZeneca a cependant annoncé avoir suspendu son essai de phase III suite à la survenue d'un effet indésirable grave chez un patient en Angleterre. Cet incident, heureusement transitoire, car l'essai a très rapidement repris, rappelle la grande difficulté d'un développement accéléré et sûr d'un vaccin. Si, néanmoins, ce vaccin fonctionne, le Serum Institute et le gouvernement indien se sont engagés à réserver une moitié du stock de vaccin à I'Inde et à en fournir une autre moitié aux pays à faibles revenus, par le biais de la fondation Global Alliance for Vaccines and Immunization $(\mathrm{GAVI})^{3}[22]$.

II résulte de ces différentes annonces, une visibilité assez floue sur les vaccins qui seront effectivement disponibles, avec des résultats scientifiques qui ne sont, actuellement, pas accessibles, notamment sur l'immunité à long terme et sur la capacité de ces vaccins à bloquer la transmission du virus. Le $1^{\text {er }}$ octobre, l'Inserm a annoncé par l'intermédiaire du projet COVIREIVAC rechercher plus de 25000 volontaires pour participer à l'évaluation des différents vaccins contre la COVID-19 [23]. Une des inconnues majeures de l'équation sera la situation épidémiologique au moment de la mise sur le marché des vaccins, puisque

\footnotetext{
${ }^{3}$ https://www.gavi.org/fr
}

l'expérience de l'épidémie de grippe HINl de 2009 nous a appris que l'acceptation d'une nouvelle vaccination est aussi très dépendante de la perception du risque épidémique par la population. Une étude récente nous apprend que l'acceptation d'un vaccin contre le SARSCoV-2 n'est, en effet, pas évidente, puisque $26 \%$ des répondeurs d'un sondage réalisé en France, disent qu'ils ne se feraient pas vacciner si une campagne était lancée [24]. Aux États-Unis, ce rejet est un sentiment partagé par $20 \%$ de la population [25]. Une majeure partie de la population mondiale reste néanmoins très attentive aux progrès réalisés pour le développement d'un vaccin anti-SARS-CoV-2 et y place beaucoup d'espoir. À côté de ces hésitants vaccinaux, des initiatives d'apprentis scientifiques pressés, comme l'initiative baptisée Rapid Deployment Vaccine Collaborative (RaDVaC), qui prône un vaccin à fabriquer soi-même (le vaccin «do it yourself »), hors de tout contrôle scientifique, démontre combien l'irrationnel a envahi le champ de la lutte contre la COVID-19 [26].

Malgré lui, le vaccin, comme d'autres approches de thérapie ou de protection anti-virales, est entré dans le champ politique, brouillant souvent le message scientifique. Le président russe a baptisé le vaccin de l'Institut Gamaleya «Spoutnik V »: un nom aux accents de guerre froide dans une déclaration qui sonne comme une démonstration de puissance à usage intérieur autant qu'extérieur. Aux États-Unis, le président, candidat à sa réélection, demande aux gouverneurs des états de l'Union à se tenir prêts à des campagnes de vaccination, dès le premier novembre... II utilise ainsi le vaccin comme un argument de campagne pour faire oublier sa gestion catastrophique de la crise. De nombreuses voix dans la communauté scientifique appellent à plus de mesure et de réalisme dans le calendrier de mise à disposition d'une vaccination disponible pour tous qui ne s'opérera vraisemblablement pas avant la fin du premier semestre 2021.

Les vaccins feront l'objet d'une mise à disposition progressive. Aussi, en France, deux rapports récents du Conseil scientifique du gouvernement sur la COVID-19 et de la Haute autorité de santé (HAS) tentent de proposer une stratégie globale de vaccination en fonction des données disponibles dans la littérature $[27,28]$; le vaccin sera distribué en priorité aux personnes exposées (personnels soignants notamment) et aux personnes fragiles. Devant les réticences face à la vaccination, il semble donc qu'un important travail d'information et de conviction reste à réaliser auprès de la population si nous souhaitons pouvoir vivre, à l'avenir, à l'abri des dangers de la COVID-19, mais aussi des autres maladies infectieuses virales qui continuent et continueront de faire des ravages dans de nombreux pays. $\diamond$ 


\section{SUMMARY}

\section{COVID-19 and vaccination: a global disruption}

Coronavirus disease (COVID)-19 is an emerging pandemic infection whose significant ability to spread in a naive population is well established. The first response of states to the COVID-19 outbreak was to impose lock-down and social barrier measures, such as wearing a surgical mask or social distancing. One of the consequences of this pandemic in terms of public health was the suspension or slowdown of infant vaccination campaigns, in almost all countries. The indirect effects of COVID-19 may therefore weigh on mortality from measles and polio in developing countries. In this pandemic chaos, the only hope lies in the rapid development of an effective vaccine against severe acute respiratory syndrome-coronavirus-2 (SARS-CoV-2). However, acceptance of this vaccine has not yet been won, as beyond the many unknowns that will inevitably weigh around such rapid development, skepticism among vaccine hesitants is growing. $\diamond$

\section{LIENS D'INTÉR}

Les auteurs déclarent n'avoir aucun lien d'intérêt concernant les données publiées dans cet article.

\section{RéFÉRENCES}

1. Avis $n^{\circ} 2020.0025 / A C / S E E S P$ du $1^{\text {er }}$ avril 2020 du collège de la Haute autorité de santé relatif au maintien de la vaccination des nourrissons dans le contexte de l'épidémie de COVID-19. Haute autorité de santé (HAS), 2020. https://www.has-sante.fr/jcms/p_3169084/fr/avis-n-2020-0025/ ac/seesp-du-ler-avril-2020-du-college-de-la-haute-autorite-de-sante-relatif-au-maintiende-la-vaccination-des-nourrissons-dans-le-contexte-de-I-epidemie-de-covid-19

2. Calendrier vaccinal des nourrissons et COVID-19. Paris : Académie Nationale de Médecine, 2020» http://www.academie-medecine.fr/wp-content/uploads/2020/04/Calendrier-vaccinal-desnourrissons-et-COVID-19.pdf

3. Weill A, Drouin J, Desplas D, et al. Usage des médicaments de ville en France durant l'épidémie de Covid-19 : point de situation après 5 semaines de confinement. Étude pharmaco-épidémiologique à partir des données de remboursement du SNDS. EPIPHARE rapport 2. Paris : ANSM, 2020. https://ansm.sante.fr/S-informer/Points-d-information-Points-d-information/Usage-desmedicaments-en-ville-durant-I-epidemie-de-Covid-19-point-de-situation-apres-cinqsemaines-de-confinement-Point- $d$-information

4. Check Hayden $\varepsilon$. Ebola obstructs malaria control. Nature $2014 ; 514: 15-6$.

5. Nelson R. COVID-19 disrupts vaccine delivery. Lancet Infect Dis $2020 ; 20: 546$.

6. Macklin GR, O'Reilly KM, Grassly NC, et al. Evolving epidemiology of poliovirus serotype 2 following withdrawal of the serotype 2 oral poliovirus vaccine. Science $2020 ; 368: 401-5$.

7. Roberts L. Polio vaccinators are back after pandemic pause. Science $2020 ; 369: 360$

8. Guiding principles for immunization activities during the COVID-19 pandemic. Interim guidance. Genève : WHO, 2020.

9. Guha-Sapir D, Moitinho de Almeida M, Keita M, et al. COVID-19 policies: remember measles. Science $2020 ; 369: 261$.
10. Roberton T, Carter ED, Chou VB, et al. Early estimates of the indirect effects of the COVID-19 pandemic on maternal and child mortality in low-income and middleincome countries: a modelling study. Lancet Glob Health 2020 ; 8 : e901-e8.

11. Le T, Cramer JP, Chen R, Mayhew S. Evolution of the COVID-19 vaccine development landscape. Nat Rev Drug Discov 2020 ; 19 :667-8.

12. Folegatti PM, Ewer KJ, Aley PK, et al. Safety and immunogenicity of the ChAdOxl nCoV-19 vaccine against SARS-CoV-2: a preliminary report of a phase 1/2, single-blind, randomised controlled trial. Lancet $2020 ; 396: 467-78$.

13. Zhu FC, Guan XH, Li YH, et al. Immunogenicity and safety of a recombinant adenovirus type-5-vectored COVID-19 vaccine in healthy adults aged 18 years or older: a randomised, double-blind, placebo-controlled, phase 2 trial. Lancet $2020 ; 396: 479-88$.

14. Logunov DY, Dolzhikova IV, Zubkova OV, et al. Safety and immunogenicity of an rAd26 and rAd5 vector-based heterologous prime-boost COVID-19 vaccine in two formulations: two open, non-randomised phase $1 / 2$ studies from Russia. Lancet 2020 ; doi.org/10.1016/S0140-6736(20)31866-3-

15. Abbott $A$. Researchers highlight questionable data in Russian coronavirus vaccine trial results. Nature $2020 ; 585: 493$.

16. Keech C, Albert G, Cho I, et al. Phase 1-2 trial of a SARS-CoV-2 recombinant Spike protein nanoparticle vaccine. N Engl J Med 2020 ; Doi:10.1056/ NEJMoa2026920.

17. Jackson LA, Anderson $\varepsilon$ J, Rouphael NG, et al. An mRNA vaccine against SARS-CoV-2 - preliminary report. N Engl J Med 2020 ; doi: 10.1056/ NEJMoa2022483.

18. Pitard B. Nanotaxi® pour les vaccins ARN et ADN. Med Sci (Paris) $2019 ; 35$ : 749-52

19. Mulligan MJ, Lyke KE, Kitchin N, et al. Phase $1 / 2$ study of COVID-19 RNA vaccine BNT162bl in adults. Nature $2020 ; 12$ August.

20. Sahin U, Muik A, Derhovanessian $\varepsilon$, et al. COVID-19 vaccine BNT162bl elicits human antibody and $\mathrm{T}_{H} \mathrm{l}$ T-cell responses. Nature $2020 ; 30$ September.

21. Bar-Zeev N, Moss WJ. Encouraging results from phase $1 / 2$ COVID-19 vaccine trials. Lancet $2020 ; 396: 448-9$.

22. Vaidyanathan $G$. India will supply coronavirus vaccines to the world: will its people benefit? Nature 2020 ; doi: 10.1038/d41586-020-02507-x.

23. https://www.covireivac.fr

24. Group C. A future vaccination campaign against COVID-19 at risk of vaccine hesitancy and politicisation. Lancet Infect Dis $2020 ; 20: 769-70$.

25. Expectations for a COVID-19 Vaccine. Chicago : Associated Press-NORC Center for Public Affairs Research, 2020.

26. Caplan AL, Bateman-House AL. The danger of DIY vaccines. Science 2020 ; $369: 1035$

27. Stratégie de vaccination contre le COVID-19. Anticipation des scénarios possibles de vaccination et recommandations préliminaires sur les populations cibles. Saint-Denis : Haute Autorité de Santé (HAS), 2020. https://www.has-sante.fr/jcms/p_3197106/fr/strategie-de-vaccinationcontre-le-covid-19-anticipation-des-scenarios-possibles-de-vaccinationet-recommandations-preliminaires-sur-les-populations-cibles

28. Barré-Sinoussi F, Atlani-Duault L, Muller S, et al. Vaccins contre le SARS-CoV-2. 9 juillet 2020. Une stratégie de vaccination. Paris : Ministère des solidarités et de la santé, 2020. https://solidarites-sante.gouv.fr/IMG/pdf/avis_vaccins_9_ juillet_2020_-_care_-_conseil_scientifique_-_comite_vaccin.pdf

\section{TIRÉS À PART}

J.N. Tournier

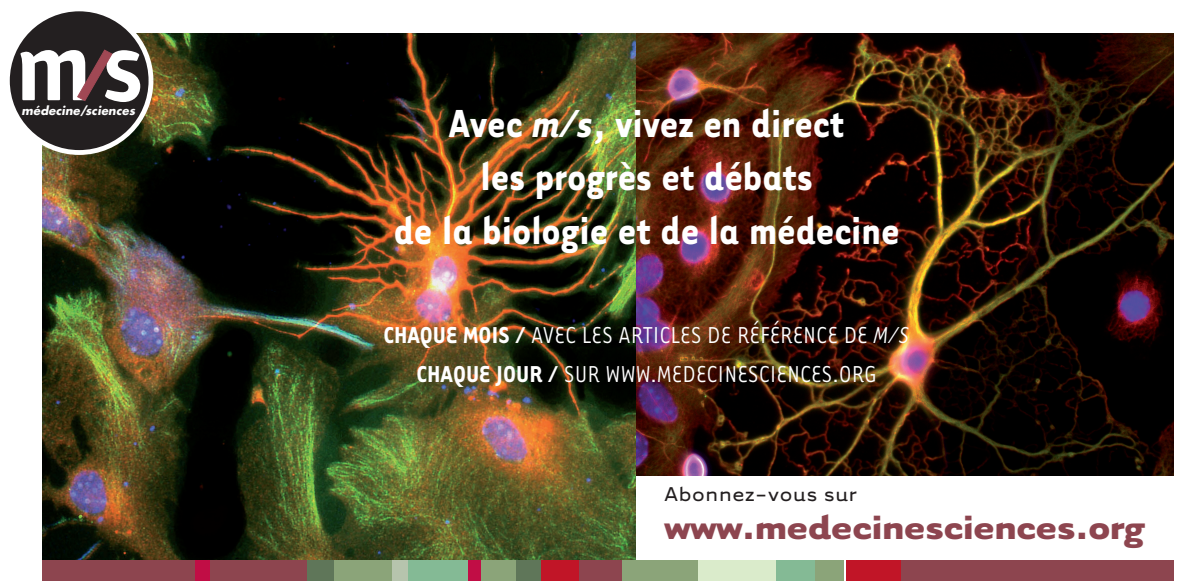

